\title{
Fabrication and Magnetotransport Properties of Carbon Films with Embedded Metal Nanoclusters
}

\author{
V.K. Ksenevich ${ }^{a, *}$, I.A. BashmaKov ${ }^{b}$, A.A. Melnikov ${ }^{c}$ \\ AND A.D. WIECK ${ }^{c}$ \\ ${ }^{a}$ Laboratory of Physics of Electronic Materials, Department of Physics \\ Belarus State University, F. Skaryna av. 4, 220050 Minsk, Belarus \\ ${ }^{b}$ Department of Chemistry, Belarus State University \\ F. Skaryna av. 4, 220050 Minsk, Belarus \\ ${ }^{c}$ Department of Physics and Astronomy, Bochum Ruhr-University \\ Universitaetstr., 150, 44780 Bochum, Germany
}

\begin{abstract}
2-dimensional arrays of $\mathrm{Co}$ - and $\mathrm{Pd}$-clusters embedded in carbon films were fabricated by means of heat-treatment method of carboxylated cellulose films after the exchange of $\mathrm{COOH}$-group protons by $\mathrm{Co}-$ and Pd-cations. The sizes of metal clusters within range $10 \mathrm{~nm}-1 \mu \mathrm{m}$ were obtained in dependence on the heat-treatment temperature. The dependencies of the resistance on temperature and magnetic field for the samples annealed at $T=700^{\circ} \mathrm{C}$ and $900^{\circ} \mathrm{C}$ were measured. The $R(T)$ dependencies both for carbon films with Co- and Pd-clusters can be fitted by expression $R=R_{0} \exp \left(T_{0} / T\right)^{1 / n}$ inherent for variable-range hopping. In the whole range of investigated magnetic field and temperature magnetoresistance was negative and can be related to quantum interference in the variable range hopping transport along neighboring alternative paths.
\end{abstract}

PACS numbers: 61.46.+w, 73.63.-b, 75.47.-m

\section{Introduction}

Nanoscale metallic particles are known to exhibit unusual chemical and electronic properties $[1,2]$. Monolayers of magnetic nanoclusters are promising materials for magnetic sensors and magnetic storage media with a high storage density. Arrays of noble metal nanoclusters can be used to study single-electron tunneling phenomena. Different techniques are currently used for the fabrication of

*corresponding author; e-mail: ksenevich@bsu.by 
2-dimensional arrays of metal clusters with nanoscale sizes: ion etching of metal films through arrays of polymer nanoparticles fabricated by self-assembling using direct surface adsorption or through the Langmuir-Blodgett technique [3, 4], deposition of island-like metal films using sputtering [5], ion-beam metal cluster preparation technique [6]. We propose a new simple method for fabrication of 2-dimensional arrays of magnetic nanoclusters.

\section{Experimental}

In order to obtain Co- and Pd-clusters embedded in carbon matrix, the heat-treatment method of carboxylated cellulose films after the exchange of $\mathrm{COOH}$ -group protons by cobalt and palladium cations by means of ion-exchange adsorption was employed. The homogeneous distribution of cations on atomic scale in polymer film as a result of ion-exchange adsorption is expected to give opportunity to obtain ordered arrays of Co- or Pd-clusters within the carbon film after heat treatment. The carbon fibers with embedded Co-nanoclusters were earlier obtained by Bashmakov et al. [7] using this technique. In our study thin polymer films instead of rayon fibers were used as precursors in order to obtain 2-dimensional arrays of metal nanoclusters. For the fabrication of polymer precursors a droplet of the complex liquid - diluted carboxylated cellulose solution (amyl acetate with ethyl alcohol was used as a solvent) - was deposited on the surface of water. Thin polymer film was fabricated after spreading of carboxylated cellulose solution. After the evaporation of the solvent, the films were transferred onto isolating substrates for further ion-exchange absorption and heat-treatment process. Co and Pd cations were introduced into cellulose films from water solution of cobalt and palladium acetates, respectively, similar to the procedure proposed for the carbon fibres [7]. Carboxylated cellulose with content of $\mathrm{COOH}$-groups in the range 4.3-16.1 wt\% was used. Then polymer films with metal cations were annealed in vacuum at a residual pressure of about $1 \mathrm{~Pa}$. Temperature rising rate was of about $10^{\circ} \mathrm{C} / \mathrm{min}$. Samples were isothermally annealed at different temperature $\left(350^{\circ} \mathrm{C}, 700^{\circ} \mathrm{C}, 900^{\circ} \mathrm{C}\right)$ during $30 \mathrm{~min}$ in order to stabilize thermochemical conversions. Finally, after heat-treatment process carbon films with embedded cobalt and palladium clusters were obtained.

\section{Results and discussions}

The size, structure, and distribution of the metal clusters in carbon films were investigated by means of transmission electron microscopy (TEM) and atomic force microscopy (AFM). On the basis of analysis of TEM and AFM images it was found that after heat treatment process metal clusters in carbon films were fabricated. The influence on the clusters sizes and their distribution of heat treatment temperature, concentration of the polymer solution, content of the carboxyl groups in 

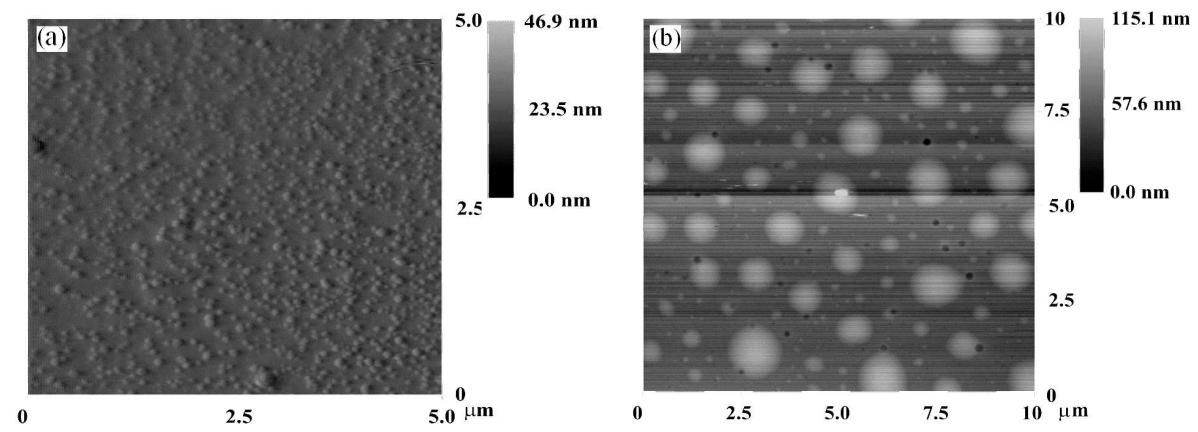

Fig. 1. AFM images of cobalt clusters embedded in carbon films for two temperatures of heat-treatment: (a) $\mathrm{T}=350^{\circ} \mathrm{C}$, (b) $\mathrm{T}=700^{\circ} \mathrm{C}$.

the solution and thickness of the polymer film was investigated. The cluster sizes and their distribution were found to depend mainly on the temperature of the heat treatment. Clusters with average size in the range $10-50 \mathrm{~nm}$ in dependence on the thickness of the polymer film and content of $\mathrm{COOH}$-groups were fabricated at the heat treatment at $350^{\circ} \mathrm{C}$, as shown in Fig. 1a. Increase in the heat-treatment temperature induces a rise of the size of clusters and inhomogeneity in their distribution. As shown in Fig. $1 \mathrm{~b}$ at the heat treatment at $700^{\circ} \mathrm{C}$ the clusters with sizes from some tens of nm up to about $1 \mu \mathrm{m}$ were obtained.

The transport properties of the carbon films with metal clusters embedded were measured using standard four-probe dc-technique. The electric properties of metal cluster monolayers were found to depend strongly on the heat-treatment temperature. The samples obtained at the heat-treatment temperature $350^{\circ} \mathrm{C}$ were found to be ohmic. Dependencies of the resistance on the temperature and magnetic field for the samples annealed at $700^{\circ} \mathrm{C}$ and $900^{\circ} \mathrm{C}$ were measured. Magnetoresistance measurements were carried out in superconducting magnet in the temperature range $20-50 \mathrm{~K}$ at magnetic fields up to $5 \mathrm{~T}$. The magnetic field was applied perpendicularly to the film plane.

The $R(T)$ dependencies both for carbon films with Co- and Pd-clusters can be fitted by expression $R=R_{0} \exp \left(T_{0} / T\right)^{1 / n}$ inherent for variable-range hopping [8]. The $R(T)$ dependencies for the samples with Co-clusters obtained at the heat-treatment temperature $700^{\circ} \mathrm{C}$ and $900^{\circ} \mathrm{C}$ are shown in Fig. 2 (curve 1 and curve 2, respectively). For the samples annealed at $700^{\circ} \mathrm{C}$ parameter $n=2$ in the whole investigated temperature range. A crossover from the Mott variable range hopping $(n=4)$ to the Coulomb-gap Efros-Shklovskii variable range hopping $(n=2)$ upon decrease in temperature was experimentally observed at $T$ of about $100 \mathrm{~K}$ for the carbon films with Co-clusters obtained at $900^{\circ} \mathrm{C}$. Similar $R(T)$ behavior was observed for mesosocopic carbon networks obtained by means of carbonization method of the self-assembled polymer periodic structures $[9,10]$.

In the whole investigated magnetic field and temperature range magnetoresistance was negative and linearly depended on magnetic field. As shown in Fig. 3, 


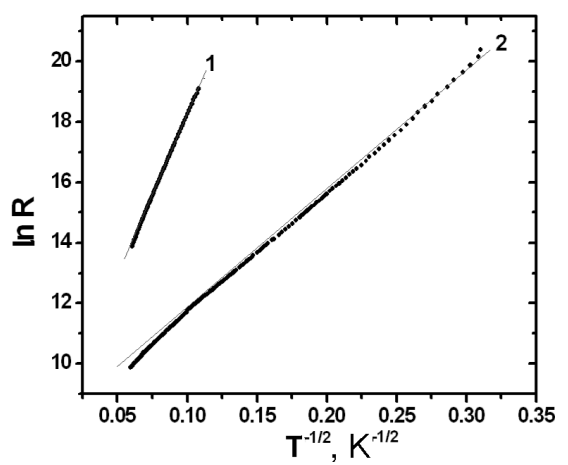

Fig. 2. The temperature dependence of the resistance of the carbon films with Co clusters obtained at the heat treatment temperature $700^{\circ} \mathrm{C}$ (curve 1) and $900^{\circ} \mathrm{C}$ (curve 2).

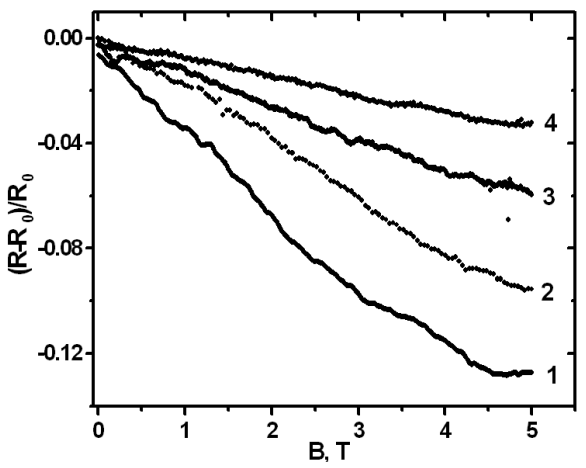

Fig. 3. The dependence of the resistance on the magnetic field for carbon films with Co clusters annealed at $900^{\circ} \mathrm{C}$ and at the temperatures $T: 1-18 \mathrm{~K}, 2-29 \mathrm{~K}, 3-$ $36 \mathrm{~K}, 4-49 \mathrm{~K}$.

with decreasing temperature, the negative magnetoresistance becomes stronger. Such magnetoresistance behavior is in a good agreement with the model proposed by Nguen et al. and related to quantum interference in the variable range hopping transport along neighboring alternative paths [11].

It should be noted that there are no essential differences between dependencies of the resistance on temperature and magnetic field for carbon films with Pdand Co-clusters obtained at the same heat treatment temperature. It means that electrical properties of the samples are determined by structural properties of the carbon matrix.

\section{Conclusion}

In conclusion, method for the preparation of thin carbon films with Co- and $\mathrm{Pd}$-clusters based on the exchange of $\mathrm{COOH}$-group protons by $\mathrm{Co}$ - and $\mathrm{Pd}$-cations after heat-treatment of carboxylated cellulose films was proposed. The sizes of 
metal clusters within range $10 \mathrm{~nm}-1 \mu \mathrm{m}$ were obtained in dependence on the heat-treatment temperature. Variable range hopping conductivity in the carbon matrix was found to be the charge transport mechanism in the carbon films with metal nanoclusters.

\section{Acknowledgments}

This work was supported by the Foundation of Basic Research of Belarus (project N MS03-054). One of us (V.K.) would like to acknowledge DAAD for financial support and Prof. A.D. Wieck for hospitality.

\section{References}

[1] J.H. Fendler, Chem. Mater. 13, 3196 (2001).

[2] G. Philipp, C. Müller-Schwanneke, M. Burghard, S. Roth, K. v. Klitzing, J. Appl. Phys. 85, 3374 (1999).

[3] T.P. Russel, Current Opinion in Colloid and Interface Science 1, 107 (1996).

[4] S. Zhu, R.J. Gambino, M.H. Rafailovich, J. Sokolov, S.A. Schwarz, R.D. Gomez, IEEE Trans. Magn. 33, 3022 (1997).

[5] H.R. Stuart, D.G. Hall, Appl. Phys. Lett. 69, 2327 (1996).

[6] B. Weitzel, A. Schreyer, H. Micklitz, Europhys. Lett. 12, 123 (1990).

[7] I.A. Bashmakov, V. Dorosinez, M. Lukashevich, A. Mazanik, T. Tihonova, T. Zabel, B. Wiedenhorst, H. Micklitz, J. Mater. Res. 16, 2832 (2001).

[8] B.I. Shklovskii, A.L. Efros, Electronic Properties of Doped Semiconductors, Springer-Verlag, Berlin 1984.

[9] V.A. Samuilov, J. Galibert, V.K. Ksenevich, V.J. Goldman, M. Rafailovich, J. Sokolov, I.A. Bashmakov, V.A. Dorosinets, Physica B 294-295, 319 (2001).

[10] V.K. Ksenevich, J. Galibert, V.A. Samuilov, Physica E 17, 389 (2003).

[11] V.L. Nguen, B.Z. Spivak, B.I. Shklovskii, Sov. Phys. JETP 62, 1021 (1985). 